\title{
Characterization and Modeling of a Highly Reliable ONO Antifuse for High-Performance FPGA and PROM
}

\author{
Liu Guozhu ${ }^{1}$, Wu Jianwei ${ }^{1}$, Yu Zongguang ${ }^{1}$, Hong Genshen ${ }^{1}$, Zheng Ruocheng ${ }^{1}$, Liu Baiqin ${ }^{1}$, \\ Wu Suzhen ${ }^{1}$, Du Tao ${ }^{2}$ \\ ${ }^{1}$ Key Laboratory of Radiation Hardened Integrated Circuit, China Electronics Technology Group Corporation No.58 Research Institute, Wuxi, \\ China \\ ${ }^{2}$ State Key Laboratory of Electronic Thin Films and Integrated Devices, University of Electronic Science and Technology of China, Chengdu, \\ China
}

\author{
Email address: \\ gzliuuestc@163.com (Liu Guozhu)
}

\section{To cite this article:}

Liu Guozhu, Wu Jianwei, Yu Zongguang, Hong Genshen, Zheng Ruocheng, Liu Baiqin, Wu Suzhen, Du Tao. Characterization and Modeling of a Highly Reliable ONO Antifuse for High-Performance FPGA and PROM. International Journal of Materials Science and Applications. Vol. 5, No. 3, 2016, pp. 169-177. doi: 10.11648/j.ijmsa.20160503.19

Received: June 25, 2016; Accepted: July 14, 2016; Published: July 19, 2016

\begin{abstract}
The characteristics and reliability of ONO (oxide-nitride-oxide) anti-fuse devices prepared basing on $0.6 \mu \mathrm{m}$ SOI CMOS process have been studied experimentally and theoretically. The intrinsic principles of ONO dielectric breakdown were investigated with tunneling and emission models. It has been found that the conduction mechanisms of ONO dielectrics under high electric field $(>10 \mathrm{MV} / \mathrm{cm})$ mainly obeys Fowler-Nordheim $(\mathrm{F}-\mathrm{N})$ tunneling and Poole-Frenkel (P-F) emission and Ohmic transport models. Meanwhile, the nitrogen depth distribution and the composition of the ONO stack films have been accurately determined using SIMS and EDX, respectively. The results indicate that the nitrogen concentration of interface between tunneling oxide and $\mathrm{N}+$ sub-silicon is higher than that of interface between top oxide and $\mathrm{N}+$ poly-silicon, which can contribute to prove the difference of top and bottom electrode interface barriers according to energy band diagrams of ONO anti-fuse devices. Besides, it is also found that the average breakdown voltage of ONO anti-fuse arrays and that of distribution decrease with increasing the number of anti-fuse cells, and the result is attributed to traps density of various areas. Moreover, the programming resistance of ONO anti-fuse cells and programming circuits decreases with increasing programming current, and the programming resistance of ONO anti-fuse cells can reach less than $200 \mathrm{ohm} /$ cont when programming current is above $5 \mathrm{~mA}$. And the life of unprogrammed $\mathrm{ONO}$ anti-fuse devices can reach more than 40 years under an electric stress of $5.5 \mathrm{v}$ at the temperature from $25^{\circ} \mathrm{C}$ to $125^{\circ} \mathrm{C}$. So, it can be concluded that the characteristics and reliability of the proposed ONO anti-fuse elements are suitable for applications in FPGA and PROM.
\end{abstract}

Keywords: Antifuse, Oxide-Nitride-Oxide, Breakdown Voltage, Programming Resistance, SOI

\section{Introduction}

Over the past years, due to the ONO anti-fuse devices exhibiting great difference in impedance before and after programming, the anti-fuse processing technology has attracted significant attention of IC designers and manufacturers, and is widely used in the area of nonvolatile memories or programmable array logic devices for radiation-hardened space environment, such as PROM, PAL, FPGA (field programmable gate arrays), and so on [1-6]. The composition, structure, electrical characteristics and reliability of ONO antifuses have been widely studied. The ONO dielectrics layer lies between a poly-silicon conductor and a heavily doped $\mathrm{N}+$ diffusion region of the base silicon wafer. Prior to programming, the anti-fuse cell tends to exhibit very high resistance (typically $>1 \mathrm{M} \Omega$ ), and the impedance may be reduced to below $1000 \Omega$ after programming under high voltage or current stress [1-4]. Under the programming voltage, the ONO sandwich melts and the base wafer grows an epitaxial "bump" into the poly-silicon in the shape of a dome. Growth of the bump shatters the ONO layer, allowing diffusion of the substrate $\mathrm{N}+$ into the poly-silicon to form a 
low-resistance path. The bump's size also makes the structure tolerant of current-induced stresses [1-4]. If anything, the high currents would increase the bump's size, strengthening the connection. Meanwhile, silicon-on-insulator (SOI) devices have superior capability in good radiation hardness, no latch-up, and high device density [7, 8]. Therefore, to realize high performance and reliability of radiation-hardened ONO anti-fuse devices for FPGA or PROM, the SOI CMOS processing technology can be applied in the ONO anti-fuse process.

In this work, the electrical properties and reliability of ONO anti-fuse devices were investigated experimentally and theoretically basing on $0.6 \mu \mathrm{m}$ SOI CMOS process. Meanwhile, the N/O/Si depth distributions and composition of the ONO stack films have been accurately determined using SIMS and EDX, respectively. Besides, in order to characterize the electrical properties and reliability of ONO anti-fuse devices, current-voltage (I-V) and time-dependent dielectric breakdown (TDDB) were used and performed.

\section{Experience}

\subsection{Preparation of the ONO Anti-fuse Samples}

In this paper, the ONO anti-fuse samples were prepared basing on $0.6 \mu \mathrm{m}$ SOI CMOS process (2Wells@2Polys@2Metals). The anti-fuse ONO dielectrics is formed as follows: At first, after the formation of CMOS on the SOITEC $\mathrm{P}<100>$ (top-Si/ bottom-oxide $=1.5 \mu \mathrm{m} / 3.0 \mu \mathrm{m}$ ), the high density of $\mathrm{N}+$ sub-silicon in $\mathrm{p}$-well is formed by arsenic and phosphorus implantation (dose: E15) and annealing at $1050^{\circ} \mathrm{C}$. Since, the tunneling oxide layer is very thin, the process of which is difficultly controlled through conventional methods. Meanwhile, in order to improve tunneling oxide/ $\mathrm{n}+$ sub-Silicon interface properties, the oxynitridation method is taken into account. Hence, bottom oxide (i.e., tunneling oxide) is thermally gown on $\mathrm{n}+$ sub-silicon by the low-pressure oxynitridation in mixture of $\mathrm{N}_{2} \mathrm{O}$ and $\mathrm{N}_{2}$ ambients (i.e., $\mathrm{N}_{2} \mathrm{O}: \mathrm{O}_{2}=1: 5$ ), in which the temperature and pressure are fixed at $820^{\circ} \mathrm{C}$ and 70 torr, respectively [9]. And following the rich-nitrigen silicon nitride is immediately deposited by the LPCVD method. The top-oxide is formed by re-oxidation of SixNy at $950^{\circ} \mathrm{C}$ (i.e., wet oxide). After that, a polysilicon layer is deposited and doped by phosphorus diffusion at $900^{\circ} \mathrm{C}$ utilizing a POCL3 source. The ONO anti-fuse structure is illustrated as Fig. 1.

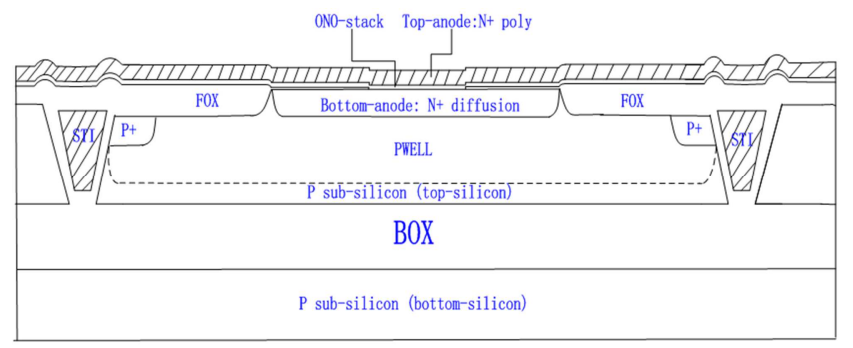

Fig. 1. Cross-sectional view of an ONO anti-fuse device basing on SOI CMOS process.

\subsection{Measurement and Method}

In this study, the cross-section of an ONO anti-fuse device and the composition of ONO dielectrics were measured by TEM and EDX, respectively, as shown in the fig. 2. The thickness of tunneling oxide and media $\mathrm{Si}_{\mathrm{x}} \mathrm{N}_{\mathrm{y}}$ and top oxide are $2.9 \mathrm{~nm} / 4.8 \mathrm{~nm} / 3.0 \mathrm{~nm}$, respectively. Meanwhile, the nitrogen depth distribution and the composition of the films have been accurately determined using SIMS, and the depth profile of $\mathrm{N} / \mathrm{O} / \mathrm{Si}$ is shown in the fig. 3. To characterize the electrical properties of ONO anti-fuse dielectrics, capacitance-voltage $(\mathrm{C}-\mathrm{V})$ and current-voltage (I-V) measurements were carried out on MOS capacitors (i.e., N+ polysilicon gate / dielectrics / N-type sub-silicon). Besides, in order to characterize the electrical properties and the reliability of ONO anti-fuse devices, I-V and time-dependent dielectric breakdown (TDDB) were used and performed. I-V and high frequency $(1 \mathrm{MHz}) \mathrm{C}-\mathrm{V}$ measurements were performed by HP model 4200 semiconductor parameter analyzer and HP model 4284 precision LCR meter, respectively.

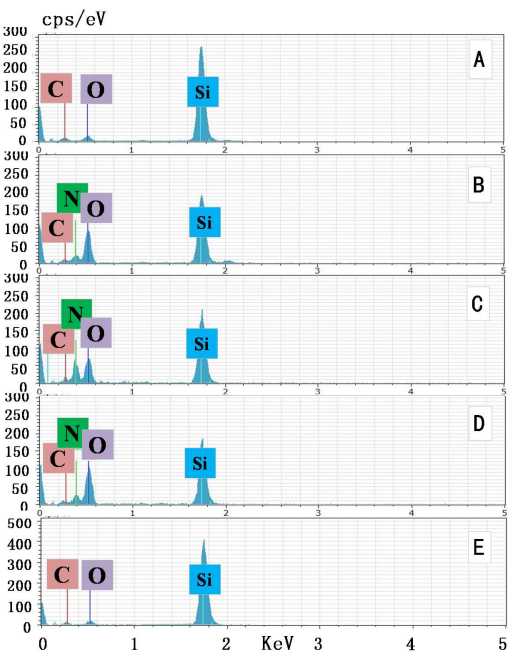

Fig. 2. TEM and EDX analysis for an ONO antifuse (A: top anode-n+polysilicon, $B|C| D$ : ONO-stack layers, E: bottom anode). 


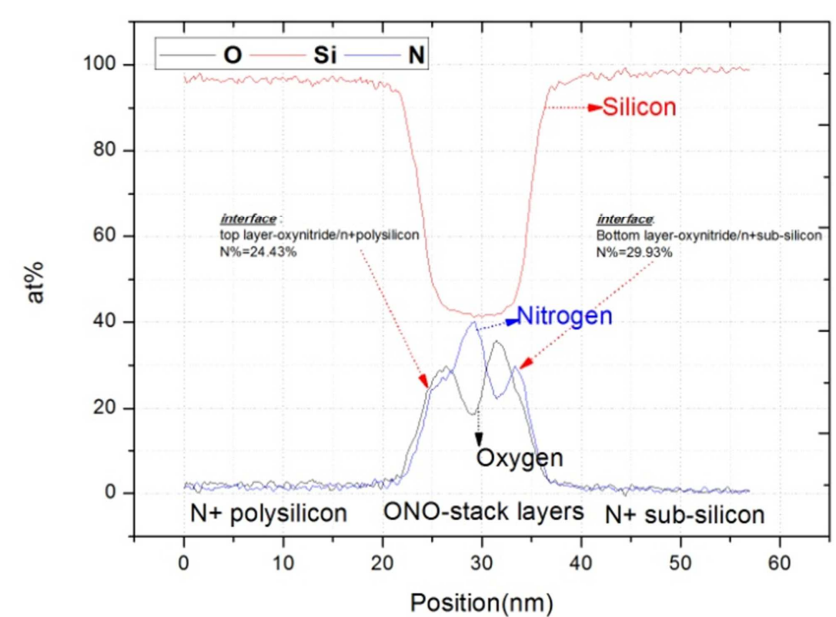

Fig. 3. SIMS depth profiles for ONO films: $N, O$, and Si distribution (sweeping from the top anode to the bottom anode).

\section{Results and Discussion}

\subsection{ONO Conduction Mechanism}

Fig. 4 shows I-V characteristics for ONO anti-fuse devices under different bias, and the effective electric field can be calculated approximately by $\mathrm{V} / \mathrm{T}_{\text {ono. }}$. It can be observed that the slopes of the I-V curves for ONO anti-fuse devices are approximately similar in the high-field region $(>7.5 \mathrm{MV} / \mathrm{cm})$ with various bias. Meanwhile, the maximum breakdown fields are also similar when $\mathrm{N}+$ sub-silicon and $\mathrm{N}+$ poly-silicon electrodes added by $+/$ - bias or $-/+$ bias, respectively. But the leakage current of low electric field is different, when $\mathrm{N}+$ sub-silicon as top polarity, the leakage current in the low electric field is higher than that of $\mathrm{N}+$ poly-silicon, and the difference has two to three orders of magnitude. Besides, the difference of breakdown voltage is approximately $2.5 \mathrm{v}$ under top and bottom electrodes with the same bias. The results can be contributed to the effect of interface roughness [10] and barrier height between cathode and ONO.

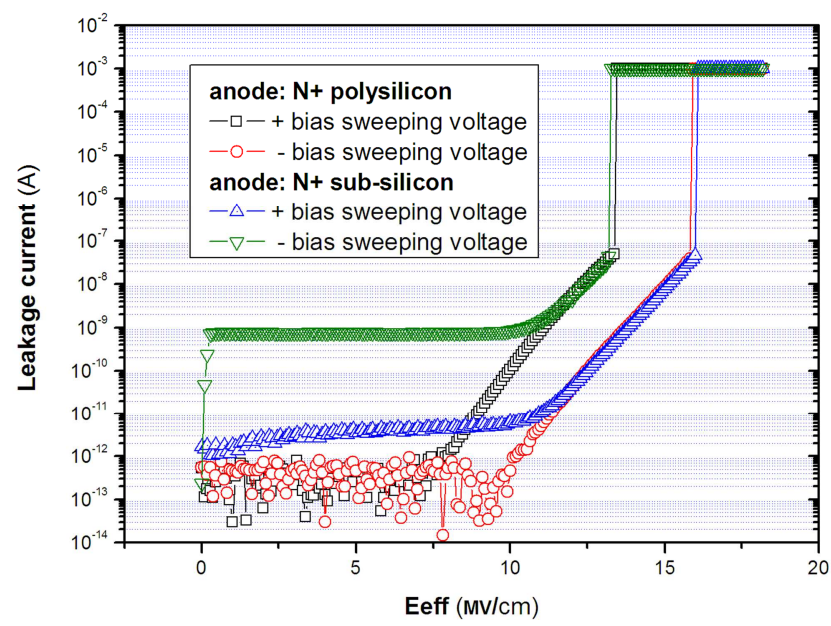

Fig. 4. I-E characteristics for the ONO structure with various bias $\left(E_{\text {eff }}=V / T_{\text {ono }}\right)$.
To study the conduction mechanism of the carriers in the ONO dielectrics, the current density (J) versus effective electric field $\left(\mathrm{E}_{\mathrm{eff}}\right)$ data were fitted by Fowler-Nordheim(F-N) tunneling, ohmic transport and Poole-Frenkel(P-F) emission models, as shown in the format (1) (5). The F-N model is based on the hot electron tunneling through a triangle-shaped energy barrier formed in the dielectric layer due to the large applied electric field. The P-F model describes the field enhanced thermal emission process of trapped electrons into the conduction band of the dielectrics [11].

$$
\mathrm{J}=\mathrm{AJ}_{1}+\mathrm{BJ}_{2}+\mathrm{CJ}_{3}
$$

Poole-Frenkel emission model:

$$
J_{1}=C_{1} E \exp \left\{-q\left[\phi_{B}-\left(q E / \pi K_{d} \varepsilon_{0}\right)^{1 / 2}\right] / k T\right\}
$$

Fowler-Nordheim tunneling model:

$$
\begin{aligned}
& J_{2}=C_{2} E^{2} \exp \left(-E_{B} / E\right) \\
& E_{B}=\frac{4(2)^{1 / 2} m^{*}\left(q \phi_{B}\right)^{3 / 2}}{3 q h}
\end{aligned}
$$

Ohmic transport model:

$$
\mathrm{J}_{3}=\mathrm{C}_{3} \mathrm{E} \exp \left(-\mathrm{E}_{\mathrm{a}} / \mathrm{kT}\right)
$$

Where $J$ is the current density, $E$ is the applied electric field, $q$ is the elementary charge, $\Phi_{\mathrm{B}}$ is the potential barrier (in zero applied electric field) that an electron must cross to move from one atom to another in the crystal, $\varepsilon_{0}$ is the dynamic permittivity, $d$ is the thickness of the insulator, $\mathrm{K}$ is Boltzmann's constant, $\mathrm{Kd}$ is dielectric constant, $T$ is the temperature, $\mathrm{C}_{1}$ is a function of the density of dielectrics trapping centers, $\mathrm{C}_{2}$ is a function of the electron effective mass and the depth of the trap-potential well, $\mathrm{C}_{3}$ is a function of the density of dielectrics trapping centers and the density of conducting carriers.

The energy band diagrams for ONO anti-fuse devices with various bias are shown in fig. 5. According to previous reports $[12,13,14]$, the ONO anti-fuse conduction mechanism can be dedicated as follows.

When $\mathrm{N}+$ polysilicon or $\mathrm{N}+$ sub-silicon is applied with a positive bias, as shown in Fig. 5(a) and (b), holes are injected into the $\mathrm{Si}_{\mathrm{x}} \mathrm{N}_{\mathrm{y}}$ film by direct tunneling through the reoxidized film (top-layer) or tunneling oxide (bottom-layer), and they are carried by the P-F mechanism in the $\mathrm{Si}_{\mathrm{x}} \mathrm{N}_{\mathrm{y}}$ film (middle-layer). The holes will accumulate at the interface of the middle-layer and the bottom oxide or the middle-layer and the top-layer, although partly holes are compensated for F-N tunneling electron. Thus, such holes lead to electric field enhancement of bottom oxide (B.O) and enhance the F-N electron conduction in B.O.

However, when $\mathrm{N}+$ polysilicon or $\mathrm{N}+$ sub-silicon electrode is added by a negative bias, as shown in Fig. 5(c) and (d), holes are injected into the valence band of the $\mathrm{Si}_{\mathrm{x}} \mathrm{N}_{\mathrm{y}}$ film by 
the modified F-N tunneling because of the thin bottom oxide film, and they are carried by the P-F mechanism. For hole conduction from the $\mathrm{Si}_{\mathrm{x}} \mathrm{N}_{\mathrm{y}}$ film to the electrode, two mechanisms are thought to occur: Tunneling from the trap site

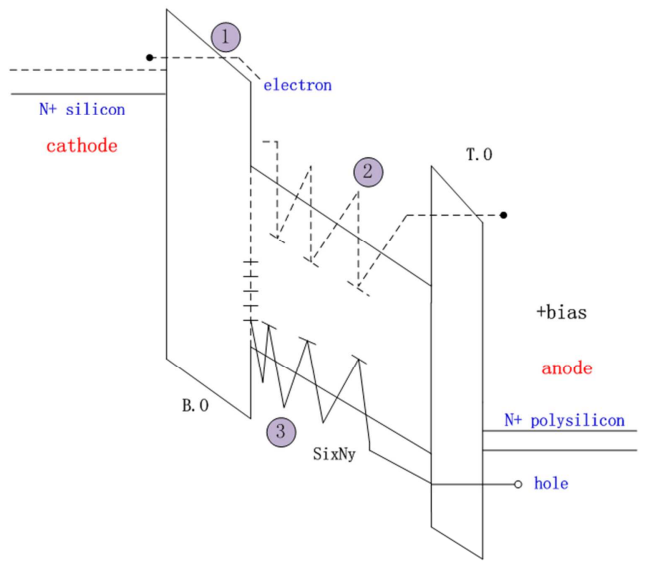

(a)

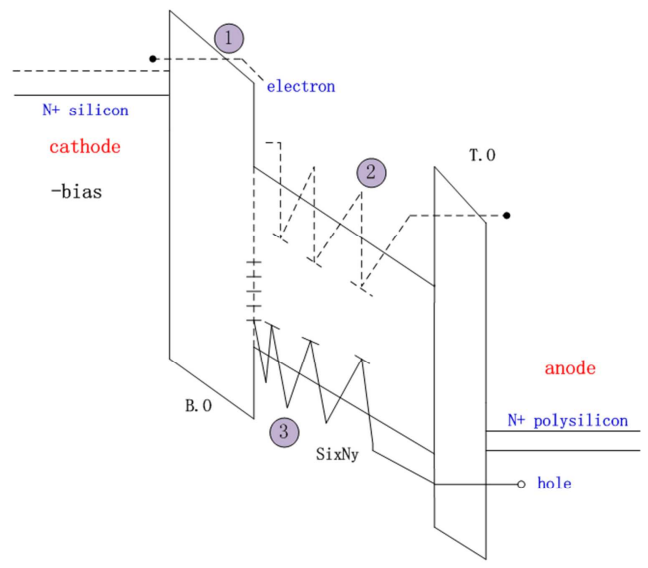

(c) of the $\mathrm{Si}_{\mathrm{x}} \mathrm{N}_{\mathrm{y}}$ film to the electrode through the oxide and tunneling from the valence band to the electrode. The tunneling conduction from the trap site is thought to be dominant.

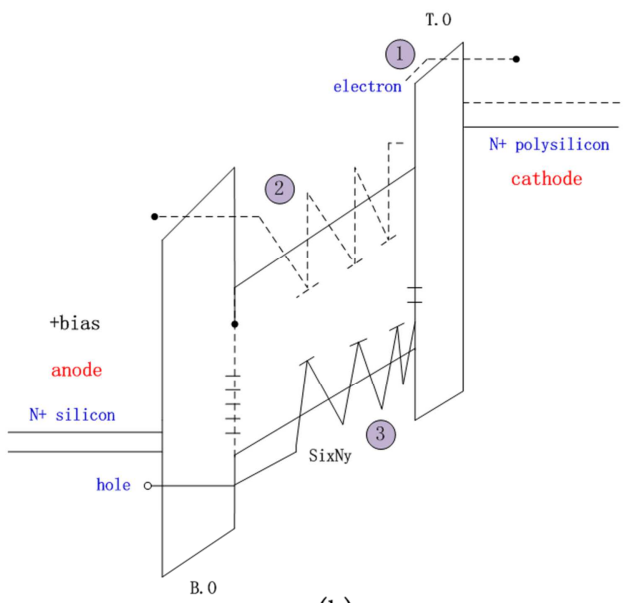

(b)

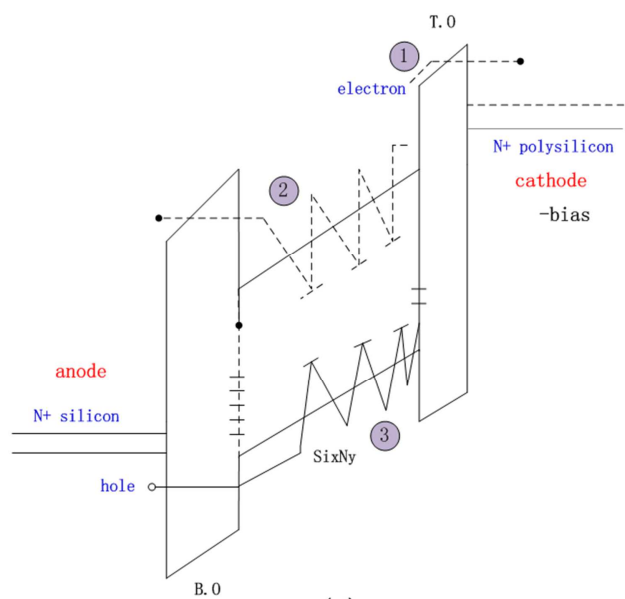

(d)

Fig. 5. The energy band diagram for ONO structure with various bias ( $a$ : $N+$ polysilicon electrode is applied with a positive sweeping voltage; $b$ : $N+$ sub-Silicon electrode is applied with a positive sweeping voltage; $c: N+$ sub-Silicon electrode is applied with a negative sweeping voltage; $d$ : $N+$ polysilicon electrode is applied with a negative sweeping voltage).

Table 1. ONO anti-fuse key stacker parameters at $25^{\circ} \mathrm{C}$ (a value are from Yu H.Y [6]).

\begin{tabular}{llll}
\hline ONO stacker & $\triangle \mathbf{E}_{\mathbf{C}}(\mathbf{e v})$ & $\triangle \mathbf{E}_{\mathbf{V}}(\mathbf{e v})$ & $\mathbf{E g}(\mathbf{e v})$ \\
\hline Top-layer & 2.31 & 2.82 & $6.25^{\mathrm{a}}$ \\
Middle-layer & - & - & $5.8^{\mathrm{a}}$ \\
Bottom-layer & 2.79 & 2.84 & $6.75^{\mathrm{a}}$ \\
\hline
\end{tabular}

As shown in the fig. 3, the SIMS results clearly demonstrate that the wet re-oxidation of $\mathrm{Si}_{3} \mathrm{~N}_{4}$ results in a high concentration of nitrogen near ONO stack layers, and the nitrogen concentration of top-oxide and middle-oxide and tunneling oxide approximately are $24.43 \%, 40.15 \%$, 29.93\%, respectively. According to Yu etal [15] results of the relation between " the electron or hole barrier height and dielectric gap energy" and "the $\mathrm{N}$ concentration of oxynitride films", the band gap energy of top-oxide and middle-oxide and tunneling oxide approximately are $6.75 \mathrm{ev}(\Delta \mathrm{Ev}$ : $3.15 \mathrm{ev} ; \Delta \mathrm{Ec}: 2.45 \mathrm{ev}$; $\Delta$ Esi: $1.12 \mathrm{ev}), 5.8 \mathrm{ev} 、 6.25 \mathrm{ev}(\Delta \mathrm{Ev}: 2.95 \mathrm{ev} ; \Delta \mathrm{Ec}: 2.15 \mathrm{ev}$; $\Delta$ Esi: $1.12 \mathrm{ev})$, as shown in the table 1.

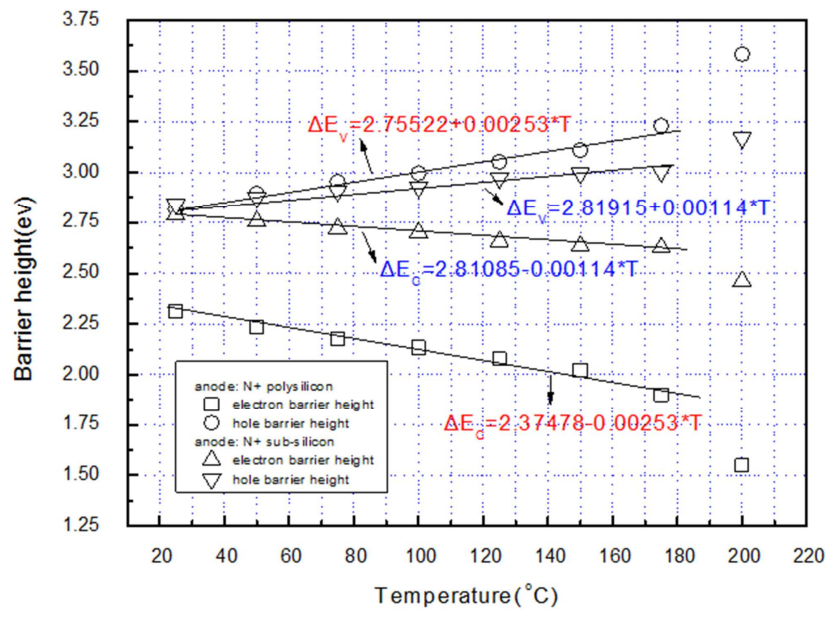

Fig. 6. The relation between interface barrier height of " tunneling oxide layer/ $N+$ sub-silicon” and " top layer oxide layer / $N+$ poly-silicon” with temperature (electron barrier height: $\Delta E_{c}$; hole barrier height: $\Delta E_{v}$ ). 
Meanwhile, the tunneling / injection properties of hot electrons (HEs) and hot holes (HHs) via the potential barrier formed by tunneling oxide or top-oxide of ONO antifuse devices are investigated. According to the principle of F-N tunneling model as shown in the format (3), the electron and hole interface barrier height of oxynitride / $\mathrm{N}+$ sub-silicon and oxynitride / $\mathrm{N}+$ poly-silicon with various temperatures for ONO anti-fuse devices are calculated basing on the slope of $\mathrm{J} / \mathrm{E}^{2}$ vs $1 / \mathrm{E}$ in different bias condition, and shown in the fig. 6 and table 1 , and that of $\Delta \mathrm{E}_{\mathrm{c}}$ and $\Delta \mathrm{E}_{\mathrm{v}}$ is in agreement with previous reports [15] at temperature $25^{\circ} \mathrm{C}$. It can be shown that the interface barrier height of the electron and hole linearly increases or decreases with temperature from $20^{\circ} \mathrm{C}$ to $180^{\circ} \mathrm{C}$, and the ONO breakdown voltage under different bias is strongly depended on the interface electron barrier height as shown in the fig. 7. It is understood that the decrease of $\Delta \mathrm{E}_{\mathrm{c}}$ exponentially enhances the tunneling probability of HEs, while it exponentially suppresses the tunneling probability of HHs. The positive charge(HHs) produced by injection is observed such that the cathode field for F-N current injection is enhanced and the anode field is decreased, depending on the location of these charge [10-18]. The trapped positive charges resulting from hole generation by impact ionization in the oxide lead to an increased local field and reduce barrier height of the cathode under high electric field. The P-F transport model suggests that electron trapping causes an increase in the electric field at the anode and eventually leads to breaking of Si-O bands [10-18].

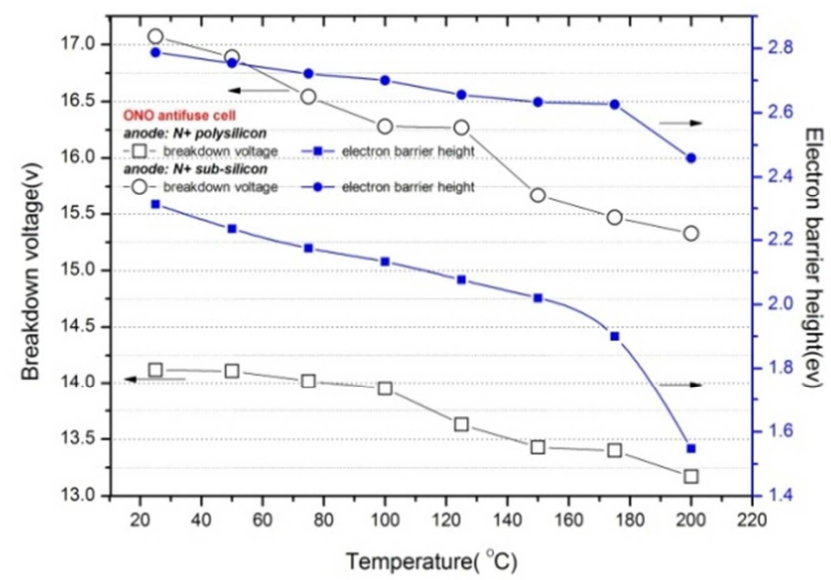

Fig. 7. ONO anti-fuse devices breakdown voltage and electron barrier height versus various temperatures from $25^{\circ} \mathrm{C}$ to $200^{\circ} \mathrm{C}$.

Fig. 8(a) and (b) show the Poole-Frenkel(P-F) and the Fowler-Nordheim(F-N) plots of the measured current-voltage relations for the $\mathrm{ONO}$ antifuse devices under various bias. The plots of $\log \left(\mathrm{J} / \mathrm{E}_{\text {eff }}\right)$ versus $\mathrm{E}_{\text {eff }}^{1 / 2}$ and $\log \left(\mathrm{J} / \mathrm{E}_{\text {eff }}^{2}\right)$ versus $\mathrm{E}_{\text {eff }}{ }^{-1}$ approximately behave in accordance with linear fitting, and that of adjective coefficients are more than 0.99 , and which indeed indicates the exactness of the model. So the ONO conduction mechanism obeys either a P-F emission model or a F-N tunneling model. Meanwhile, the dielectric constant $\mathrm{K}_{\mathrm{d}}$ can be extracted from slop of $\log \left(\mathrm{J} / \mathrm{E}_{\text {eff }}^{2}\right)$ versus $\mathrm{E}_{\text {eff }}^{-1}$ using format (2). And basing on comparing with measured $\mathrm{K}_{\mathrm{d}}$ from
$\mathrm{C}-\mathrm{V}$ characteristics of ONO anti-fuse capacitors as shown in the fig. 9 and the table 2, it is found that the matching between measured and calculated $\mathrm{K}_{\mathrm{d}}$ is very perfect, which also prove our previous conclusion. In addition, Fig. 10 shows $\log \left(\mathrm{J} / \mathrm{E}_{\mathrm{eff}}\right)$ versus $1000 / \mathrm{T}$ plots for temperatures ranging from $25^{\circ} \mathrm{C}$ to $200^{\circ} \mathrm{C}$. The temperature-dependent current at the higher electric field is mainly due to current components $\mathrm{J}_{1}$ and $\mathrm{J}_{3}$. According to the P-F emission model, current density $\mathrm{J}_{1}$ is due to field-enhanced thermal excitation of trapped electrons into the dielectric conduction band [11].

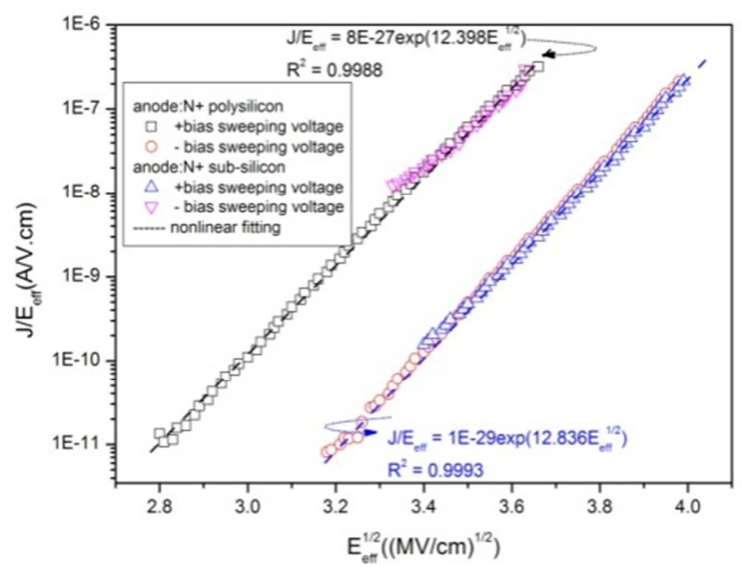

(a)

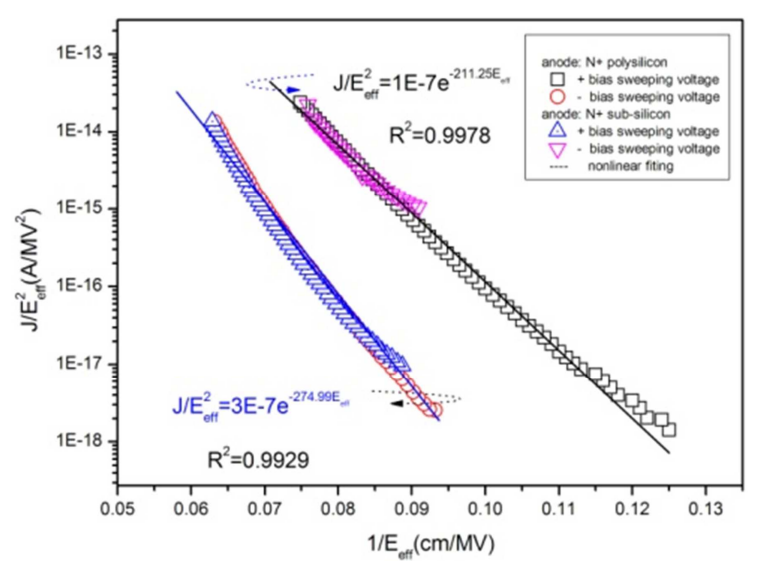

(b)

Fig. 8. Plots of (a) Poole-Frenkel: J/E eff versus $E_{\text {eff }}^{1 / 2}$, and (b) Fowler-Nordheim: $J / E_{\text {eff }}^{2}$ versus $E_{\text {eff }}^{-1}$ for $O N O$ anti-fuse devices at $25^{\circ} \mathrm{C}$.

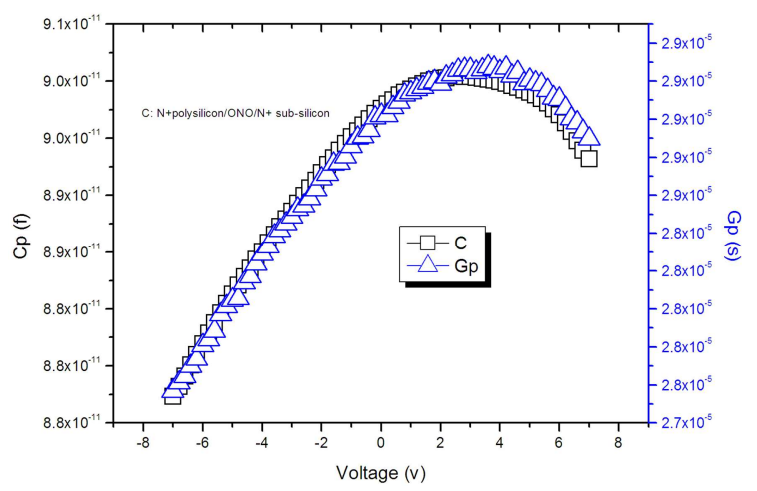

Fig. 9. C-V characteristics of ONO anti-fuse capacitors. 
Table 2. Dielectric constant (Kd) extracted from different polarities as anode and C-V measured value using ONO MOS capacitors (70 $\mu m * 300 \mu m)$.

\begin{tabular}{llll}
\hline ONO stacker & $\begin{array}{l}\text { Extracted from } \mathbf{N}+\text { polyslicon as anode } \\
\text { under positive bias }\end{array}$ & $\begin{array}{l}\text { Extracted from } \mathbf{N}+\text { sub-silicon as anode } \\
\text { under positive bias }\end{array}$ & C-V measured value \\
\hline Dielectric constant $(\mathrm{Kd})$ & 5.39 & 5.59 & 5.34 \\
\hline
\end{tabular}

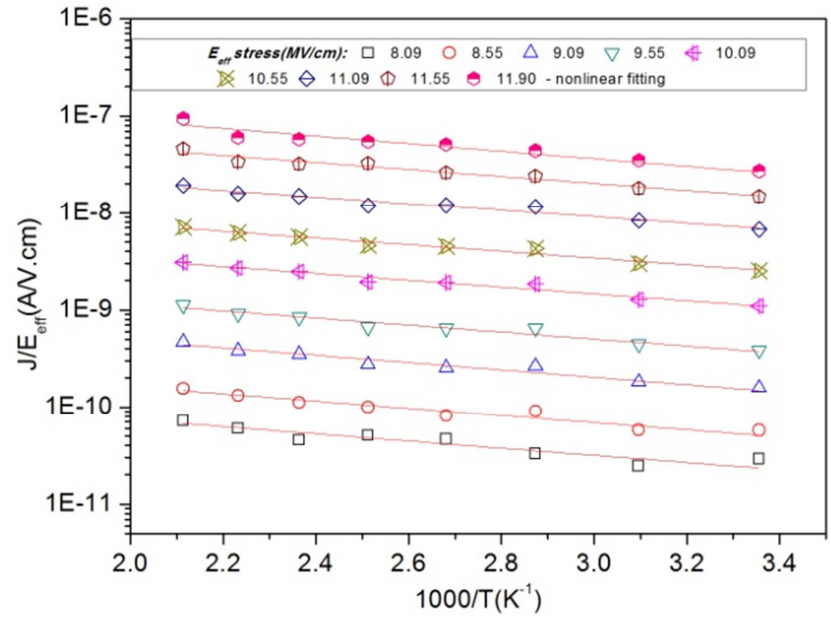

Fig. 10. $J / E_{\text {eff }}$ versus $E_{\text {eff }}^{1 / 2}$ for an $O N O$ antifuse at different electric field stresses and various temperatures from $25^{\circ} \mathrm{C}$ to $200^{\circ} \mathrm{C}$.

\subsection{ONO Characteristics}

\subsubsection{Current-Voltage Characteristics}

Fig. 11 shows excellent breakdown characteristics and distribution of ONO anti-fuse arrays containing anti-fuse cells from 1 to $1 \mathrm{~K}$. The ONO anti-fuse cells perform low leakage current under a $5.5 \mathrm{v}$ electric stress. Meanwhile, it is found that the average breakdown voltage of ONO anti-fuse arrays decreases with increasing the number of anti-fuse cells, and the distribution of breakdown voltage also decreases with increasing the number of anti-fuse cells by means of the uniformity and cumulative failure of BV, namely, the uniformity is defined as the std divided by the average of BV. The uniformity of arrays from 1 to $1 \mathrm{~K}$ decreases from $1.83 \%$ down to $0.75 \%$. The result is attributed to traps density of various areas.

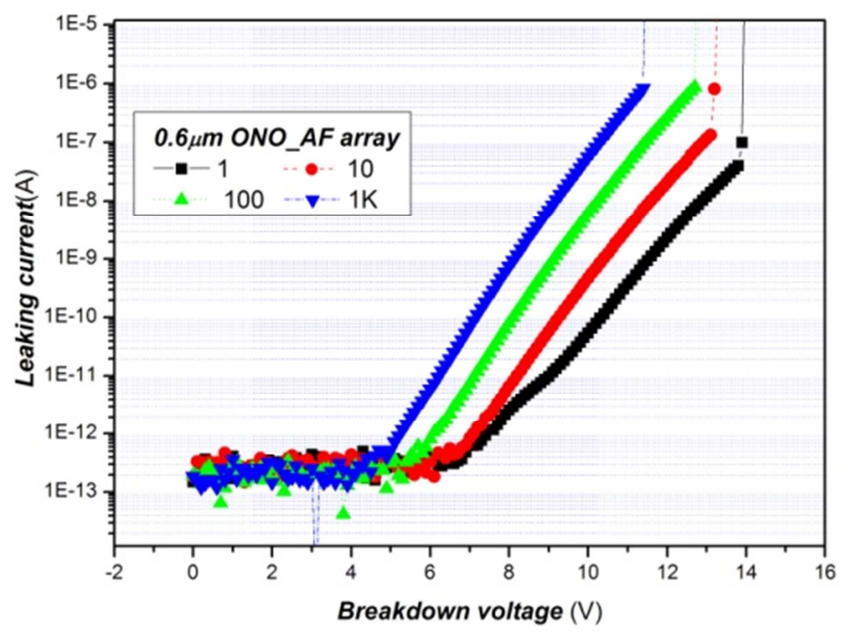

(a)

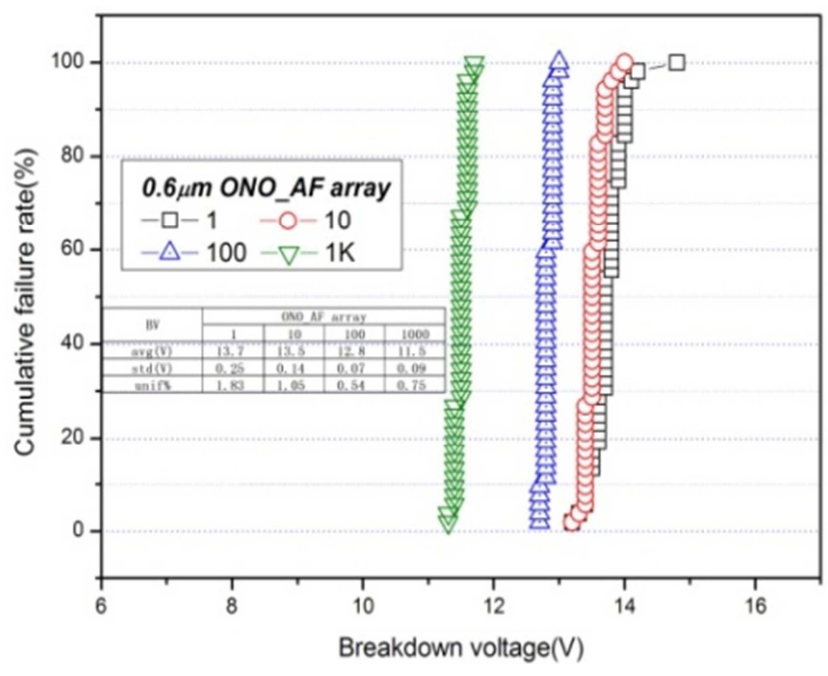

(b)

Fig. 11. The breakdown voltage and distribution of ONO anti-fuse arrays at $25^{\circ} \mathrm{C}$.

\subsubsection{Programming Characteristics}

Fig. 12 shows ONO anti-fuse programming resistance with programming time under various programming current. When programming current reaches above $0.75 \mathrm{~mA}$, the resistance of ONO anti-fuse cells becomes constant without changing under the increase of programming time, and that of characteristics further illustrates that the ONO anti-fuse cells have extremely short programming time (i.e., $\mathrm{T}_{\text {program }}=307 \mu \mathrm{s}$ ). Meanwhile, in order to study the relation between programming resistance with programming current of the ONO anti-fuse cells, we choose two testing structures: an ONO anti-fuse cell and two categories programming circuits, which contains an MOS transistor and an ONO anti-fuse cell (i.e., AF1+MOS) or an MOS transistor and two anti-fuse cells (i.e., $\mathrm{AF} 1+\mathrm{AF} 2+\mathrm{MOS}$ ). The programming resistance of $\mathrm{ONO}$ anti-fuse cells and programming circuits decreases with increasing programming current, and the programming resistance of ONO anti-fuse cells can reach less than 200 $\mathrm{ohm} /$ cont when programming current is above $2 \mathrm{~mA}$, and that of programming characteristics is shown in the fig. 13. Besides, to further illustrate the relation of programming current and resistant, the numerical analysis for the experience date is carried out as following format (6), where the factor A and B can be extracted as 948.19 and 0.7015 basing on a power fitting model, respectively.

$$
\mathrm{R}_{\mathrm{pp}}=\mathrm{A} \cdot \mathrm{I}_{\mathrm{pp}}{ }^{-\mathrm{B}}
$$




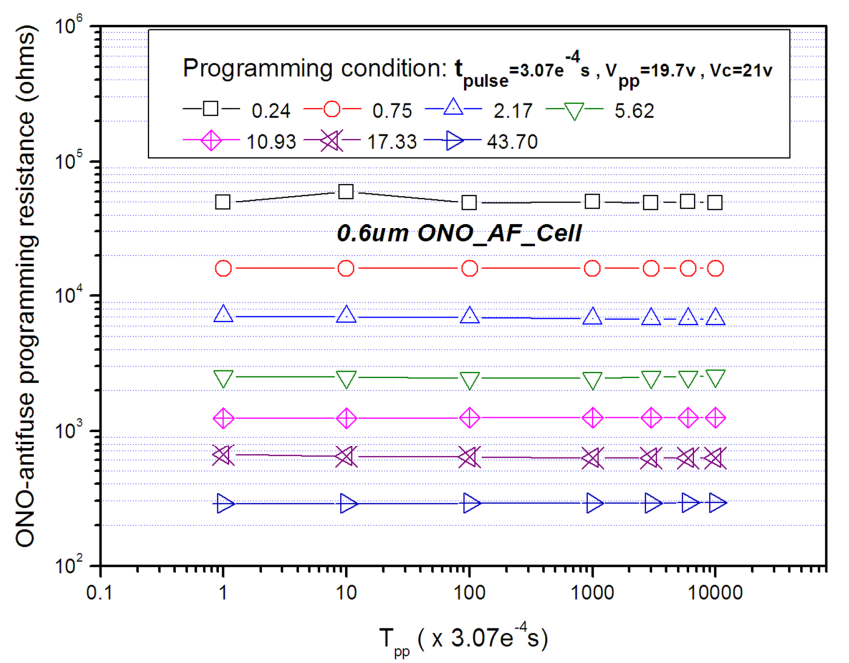

Fig. 12. ONO anti-fuse programming resistance with programming time under various programming current.

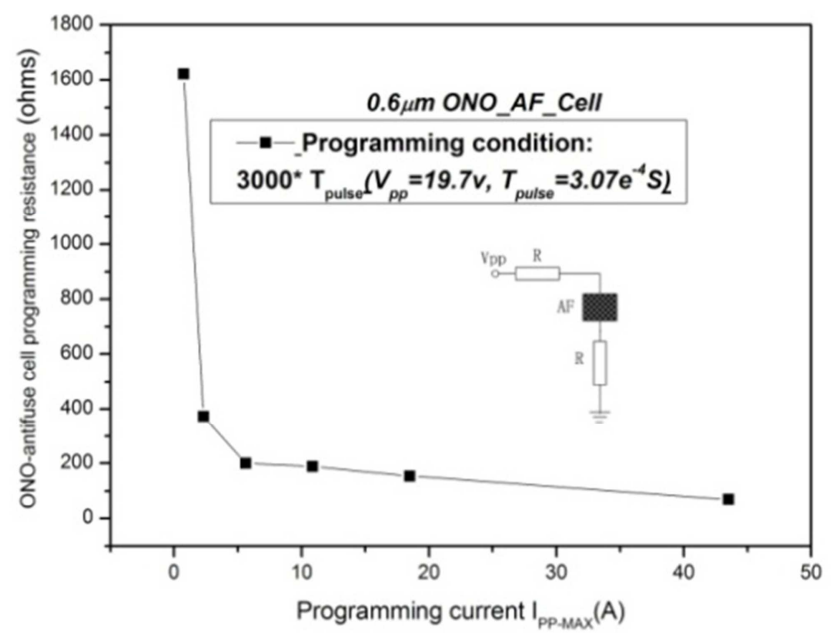

(a)

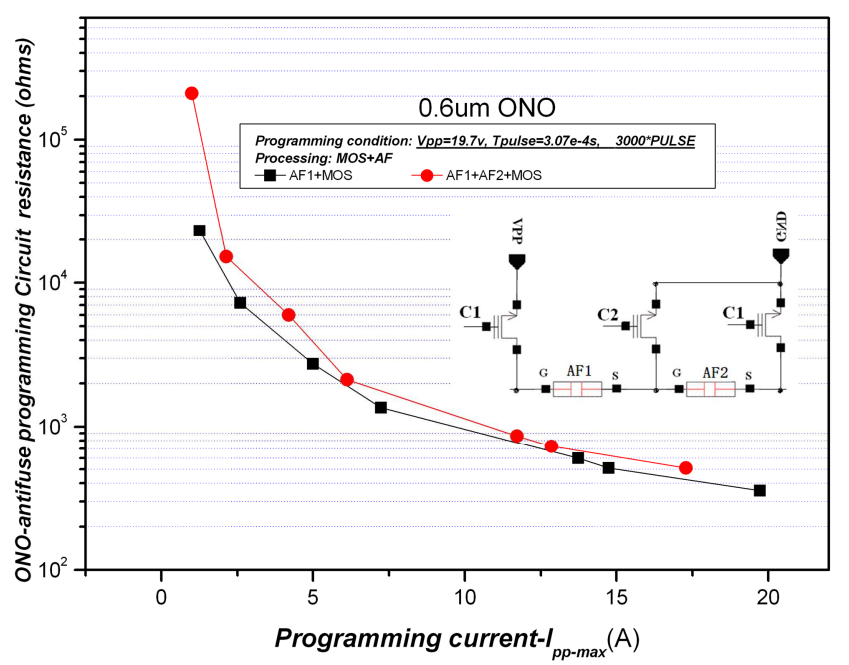

(b)

Fig. 13. ONO anti-fuse programming characteristics: (a) single anti-fuse cell; (b) programming circuits.

\subsection{Time Dependent Dielectric Breakdown (TDDB)}

According to ohmic transport model and I-V characteristics of ONO anti-fuse cells, the thermal active energy $\left(\mathrm{E}_{\mathrm{a}}\right)$ of ONO dielectrics is extracted from the slope of a $\log \left(\mathrm{J} / \mathrm{E}_{\text {eff }}\right)$ versus $\mathrm{T}^{-1}$ under high effective electric field $\left(\mathrm{E}_{\mathrm{eff}}\right)$ using the format (2) and (5), and the $E_{\text {eff }}$ is choose basing on electrode bias from $8 \mathrm{MV} / \mathrm{cm}$ to $11 \mathrm{MV} / \mathrm{cm}$ for $\mathrm{N}+$ poly-silicon as anode and from $10.5 \mathrm{MV} / \mathrm{cm}$ to $13 \mathrm{MV} / \mathrm{cm}$ for $\mathrm{N}+$ sub-silicon as anode, respectively. Meanwhile, it is also found that $E_{a}$ versus $E_{e f f}$ forms a linear equation as shown in fig. 14, and the thermal active energy $\left(E_{a}\right)$ of ONO dielectrics under a $5.5 \mathrm{v}$ electric stress condition are calculated as 0.343 and 0.349 for $\mathrm{N}+$ poly-silicon and $\mathrm{N}+$ sub-silicon as anode, respectively.

Besides, the results indeed indicate that the ONO antifuse devices conduction mechanism mainly obeys a P-F emission model or a F-N tunneling model or ohmic transport model. So the TDDB of ONO dielectrics should mainly be dependent on E model basing on Mc Pherson and Baglee ${ }^{[18,19]}$ thermochemical foundation, and the E model is expressed as an equation(7).

$$
\begin{aligned}
& \mathrm{t}_{50}=A \exp \left(-\mathrm{B} \cdot \mathrm{E}_{\mathrm{eff}}\right) \cdot \exp \left(\mathrm{E}_{\mathrm{a}} / \mathrm{kT}\right) \\
& \mathrm{E}_{\mathrm{a}}=-0.04633 \mathrm{E}_{\text {eff }}+0.57493
\end{aligned}
$$

Where $t_{50}$ is the median time to breakdown, $A$ and $B$ are constant coefficient and field acceleration parameter, respectively. According to slops of $\log \left(t_{50}\right)$ versus $E_{\text {eff }}$ under different temperature basing on the TDDB of ONO dielectrics shown in the fig. 15, the factor $\mathrm{A}$ and $\mathrm{B}$ can be extracted by $\mathrm{E}$ model (i.e., the value of $\mathrm{A}$ and $\mathrm{B}$ are about 4.38E14 and 3.3455, respectively). So, it can be calculated that the life of an ONO anti-fuse cell $\left(0.6 \mu \mathrm{m}^{*} 0.6 \mu \mathrm{m}\right)$ under an electric stress of $5.5 \mathrm{v}$ at the temperature from $25^{\circ} \mathrm{C}$ to $125^{\circ} \mathrm{C}$ is more than 40 years according to the format (7) and (8).

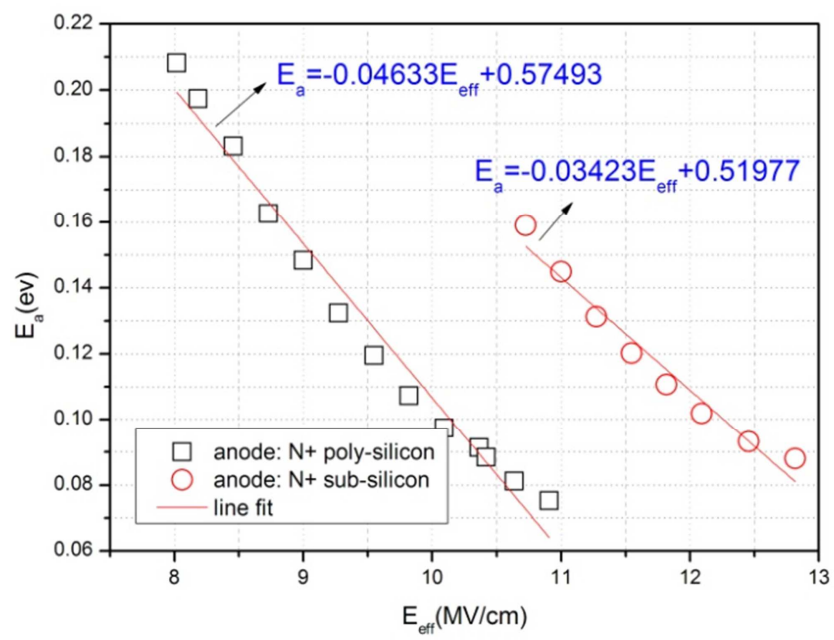

Fig. 14. Thermal active energy (Ea) versus effective electric field ( $E_{\text {eff }}$ ) for an ONO antifuse at different bias. 


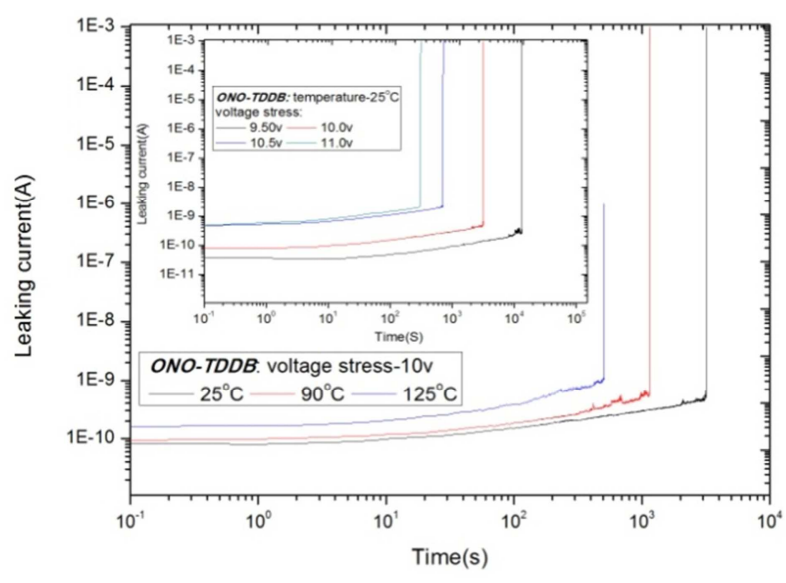

Fig. 15. TDDB of the ONO anti-fuse devices under different temperatures or electric stresses.

\section{Conclusions}

Highly reliable ONO anti-fuse devices $\left(0.6 \mu \mathrm{m}^{*} 0.6 \mu \mathrm{m}\right)$ with excellent electrical characteristics, such as low programming resistance, low programming time, and good BV uniformity, can be successfully prepared basing on $0.6 \mu \mathrm{m}$ SOI CMOS process. The intrinsic principles of ONO dielectric breakdown were investigated experimentally and theoretically with tunneling and emission models. Firstly, it has been found that the conduction mechanisms of ONO dielectrics under high electric field $(>10 \mathrm{MV} / \mathrm{cm})$ mainly obeys Fowler-Nordheim (F-N) tunneling and Poole-frenkel (P-F) emission and Ohmic transport models. Meanwhile, according to the depth profile of $\mathrm{N} / \mathrm{O} / \mathrm{Si}$ for an ONO antifuse using SIMS, the difference of top and bottom electrode interface barriers is due to the difference of nitrogen concentration at interface for "tunneling oxide $/ \mathrm{N}+$ sub-silicon" and "top oxide / N+ poly-silicon". Besides, the average breakdown voltage of ONO anti-fuse arrays and that of distribution decrease with increasing the number of anti-fuse cells, and the uniformity of arrays from 1 to $1 \mathrm{~K}$ decreases from $1.83 \%$ down to $0.75 \%$. In addition, the programming resistance of ONO anti-fuse cells and programming circuits decreases with increasing programming current, and the programming resistance of ONO anti-fuse cells can reach less than 200 ohm/cont when programming current is above $5 \mathrm{~mA}$, and the programming time Tpp is below 3.07E-4s. Finally, it can be predicated that the life of unprogrammed ONO anti-fuse devices can reach more than 40 years under an electric stress $(5.5 \mathrm{v})$ at the temperature from $25^{\circ} \mathrm{C}$ to $125^{\circ} \mathrm{C}$ by means of TDDB. So, all these results strongly indicate that the characteristics and reliability of the proposed ONO anti-fuse elements are suitable for applications in nonvolatile memory devices, such as PROM, FPGA and so on.

\section{References}

[1] S. Chiang, R. Wang, J. Chen, K. Hayes, J. McCollum, E. Hamdy, C. Hu, "Oxide-Nitride-Oxide Antifuse Reliability," International Reliability Physics Symposium, 1990, 50(12), 186-192.
[2] S. Chiang, R. Wang, T. Speers, J. McCollum, E. Hamdy, C. Hu, "Conductive channel in ONO formed by controlled dielectric breakdown," International Symposium on VLSI Technology, Systems, and Applications, 1992, 20-21.

[3] J. Chen, S. Eltoukhy, S. Yen, R. Wang, F. Issaq, G. Bakker, J. L. Yeh, E. Poon, D. Liu and E. Hamdy, "A modular $0.8 \mu \mathrm{m}$ technology for high performance dielectric antifuse field programmable gate arrays," International Symposium on VLSI Technology, Systems, and Applications, 1993, 160-164.

[4] A. Iranmanesh, Y. Karpovich, S. Yoon, "Antifuse reliability and link formation models," International Integrated Reliability Workshop, 1994, 90-94.

[5] M. M. Amr, Z. H. Esmat, L. M. John, "Programmable Low Impedance Anti-fuse Element," U.S. Pat. No. 4943538, 1990.

[6] L. M. John, "Method of forming an antifuse element with substantially reduced capacitance using the locos technique," U.S. Pat. No. 5057451, 1991.

[7] B. K. James, L. Shih-Chia, "Low-voltage SOI CMOS VLSI Devices and Circuits," John Wiley \& Sons, Inc., New York. 2001, 1-7.

[8] J. R. Schwank, V. Ferlet-Cavrois, M. R. Shaneyfelt, P. Paillet, P. E. Dodd, "Radiation Effects in SOI Technologies," IEEE TRANSACTIONS ON NUCLEAR SCIENCE, 2003, 50(3), 522-528.

[9] G. Z. Liu, G. S. Hong, R. C. Zheng, X. D. Wu, and W. J. Yang, "High Quality of Ultra-Thin SiOxNy Films Prepared in Nitrous Oxide Ambients Using Thermal Low-Pressure Oxynitridation," Materials and Manufacturing Processes, 2014, 29(9), 1050-1055.

[10] N. Matsuo, H. Fujiwara, and T. Koyanagi. "Numerical analysis for conduction mechanism of thin oxide-nitride-oxide films formed on rough poly-Si," IEEE ELECTRON DEVICE LETTERS, 1996, 17(2), 56-58.

[11] S. S. Gong, M. E. Burnham, N. D. Theodore, and D. K. Schroder. "Evaluation of Qbd for Electrons Tunneling from the $\mathrm{Si} / \mathrm{Si} 02$ Interface Compared to Electron Tunneling from the Poly-Si/Si02 Interface," IEEE TRANSACTIONS ON ELECTRON DEVICES, 1993, 40(7), 1251-1257.

[12] S. Mori, E. Sakagami, H. Araki, Y. Kaneko, K. Narita, Y. Ohshima, N. Arai, and K. Yoshikawa, "ONO Inter-Poly Dielectric Scaling for Nonvolatile Memory Applications," IEEE TRANSACTIONS ON ELECTRON DEVICES, 1991, 38(2), 386-391.

[13] Z. H. Liu, P. T. Lai, Y. C. Cheng, "Characterization of Charge Trapping and High-Field Endurance for 15-nm Thermally Nitrided Oxides," IEEE TRANSACTIONS ON ELECTRON DEVICES, 1991, 38(2), 344-354.

[14] N. Matsuo, A. Sasaki, "Electrical Characteristics of Oxide-Nitride-Oxide Films Formed on Tunnel-Structured Stacked Capacitors," IEEE TRANSACTIONS ON ELECTRON DEVICES, 1995, 42 (1), 1340-1343.

[15] H. Yu, Y. T. Hou, M. F. Li, D. L. Kwong, "Investigation of Hole-Tunneling Current Through Ultrathin Oxynitride/Oxide Stack Gate Dielectrics in p-MOSFETs," IEEE TRANSACTIONS ON ELECTRON DEVICES, 2002, 49 (7), 1158-1164.

[16] M. Wang, J. Huang, A. Anopchenko, D. Li, D. Yang, L. Pavesi, "Light emission properties and mechanism of low-temperature prepared amorphous SiNX films. II. Defect states electroluminescence," JOURNAL OF APPLIED PHYSICS, 2008, 104(8), 083505-083505-4. 
[17] Y. Y. Chiu, B. J. Yang, F. H. Li, R. W. Chang, W. T. Sun, et al, "Characterization of the charge trapping properties in p-channel silicon-oxide-nitride-oxide-silicon memory devices including SiO,” Japanese Journal of Applied Physics, 2015, 54(10), 181-184

[18] J. W. McPherson, D. A. Baglee, “Acceleration factors for thin gate oxide stressing," Microelectronics Reliability, 1986, 26(4), 796-800.

[19] J. W. McPherson, D. A. Baglee, "Acceleration factors for thin oxide breakdown," J. Electrochem. Soc., 1985, 132(8), 1903-1908. 